\title{
Entregas tardías o incorrectas en el autotransporte de carga y su relación con las condiciones laborales de los choferes: Un modelo de regresión logística Late or inaccurate road freight deliveries and their relationship to working conditions of drivers: A model of logistic regression
}

\author{
Luis David Berrones Sanz ${ }^{1}$, Patricia Cano Olivos ${ }^{2}$, Diana Sánchez Partida² y José Luis Martínez \\ Flores $^{2}$
}

Palabras Clave: autotransporte de carga; cadena de suministro; condiciones laborales de los conductores; regresión logística; entregas a tiempo

Keywords: driver trucking; supply chain; working conditions; logistic regression binary; on-time delivery

Recepción: 11-07-2017 / Aceptación: 07-03-2018

\section{Resumen}

Introducción: Los conductores del autotransporte de carga son un factor clave para satisfacer la eficiencia y la competitividad de las cadenas de suministro, por lo que están sometidos a exigencias que propician fuertes presiones derivadas de sus condiciones de trabajo. Sin embargo, no se tiene claramente identificado en qué medida sus condiciones laborales afectan la eficiencia de sus actividades. Este trabajo busca identificar en qué medida las condiciones laborales de los conductores influyen en el incumplimiento de las entregas a tiempo y, por lo tanto, repercuten en la competitividad de las cadenas de suministro.

Método: Se analizaron datos relacionados a 26,312 embarques que, en dos años, realizó una empresa de manufactura, que domina $80 \%$ de las ventas del mercado de sistemas de construcción ligera en México y que, por causas atribuidas al transportista, fueron calificados como entrega correcta o incorrecta. Debido a la naturaleza binaria de esta variable, se aplicó una regresión logística a los datos relacionados con el cumplimiento de embarques, para examinar la contribución de las condiciones laborales a los incumplimientos en las entregas a tiempo.

Resultados: El modelo generado a través de la regresión logística ofrece una precisión global de la muestra de $96.3 \%$ en la predicción del evento de incumplimiento de entrega en los embarques. De once variables independientes obtenidas de los embarques realizados, cinco -la insatisfacción, el nivel de estrés, el tiempo de tránsito, el tipo de vehículo y el tener seguridad social- se encontraron significativamente asociadas con el incumplimiento en las entregas a tiempo.

Discusión o Conclusión: Los resultados muestran una herramienta prometedora para proporcionar interpretaciones significativas que pueden utilizarse para futuras mejoras en el

\footnotetext{
${ }^{1}$ Universidad Autónoma de la Ciudad de México (UACM), México. E-mail: luis.berrones@uacm.edu.mx ${ }^{2}$ Universidad Popular Autónoma del Estado de Puebla (UPAEP), Puebla

(C) Universidad De La Salle Bajío (México)
} 
desempeño de las empresas del autotransporte de carga. Asimismo, se demuestra la importancia de las condiciones laborales y la forma en que influyen en los incumplimientos de las entregas a tiempo.

\begin{abstract}
Introduction: Cargo drivers are essential to maintain the efficiency and competitiveness of the supply chain, which subjects them to demands and strong pressures derived from their working conditions. However, it is not clear how their working conditions affect the efficiency of their activities. The objective of this work is to identify how the working conditions of cargo drivers influence on-time delivery of shipments and thus impact the competitiveness of the supply chain. Methodology: 26,312 shipments over a period of two years were analyzed. These shipments were managed by a manufacturing company with an $80 \%$ market share in light construction systems in Mexico. The shipments were identified as correct or incorrect delivery caused by the driver. Due to the binary nature of this variable, a logistic regression was applied to the data, in order to analyze how working conditions were related to the non-compliance of on-time delivery events.
\end{abstract}

Results: The model generated through the logistic regression provided a 96.3 percent global precision of the sample in predicting the failed delivery of the shipment. Eleven independent variables were obtained from the analyzed shipments, and five of those variables -dissatisfaction, level of stress, time of transit, type of vehicle, and medical coverage- were strongly associated with the inability of drivers to deliver the cargo on time.

Discussion or Conclusions: Results show a promising tool to provide meaningful interpretations that may be used for future improvements in the development of cargo and freight companies. Results also show the importance of working conditions and the way they influence the compliance of on-time delivery.

\title{
Introducción
}

En México, cada año se mueven más de 622 millones de toneladas de carga, y cerca de $83 \%$ se mueven por carretera (IMT, 2015). Así, la estrategia de transporte es una de las actividades fundamentales de la planeación logística (Ballou, 2004); y bajo este contexto, el transporte debe 
ser un servicio de calidad en términos de regularidad, oportunidad, entrega a tiempo y eficiencia; y los choferes son los agentes claves para lograr estos atributos (Berrones, 2017).

Los choferes, además de entregar las mercancías en tiempo y forma, deben realizar otras actividades derivadas de la competitividad y polivalencia del trabajo, por lo que se exponen a riesgos y exigencias derivadas de la organización y división técnica de la actividad de conducir, que los somete a condiciones laborales inadecuadas, sin seguridad social, con ritmos intensos de trabajo y muchas horas continuas de conducción, que no sólo se manifiestan en la insatisfacción y el estrés laboral y personal, sino que determinan su perfil patológico, tanto físico como mental.

Así, a pesar de que los choferes son un eslabón decisivo para el cumplimiento de los objetivos estratégicos de la cadena de suministro, con exactitud, se desconoce en qué forma las condiciones laborales de los choferes afectan las entregas correctas de los embarques. Para medir el desempeño en las entregas, la literatura sobre cadena de suministro (Chopra \& Meindl, 2016), incluye indicadores y medidas recurrentes como las entregas a tiempo (On-Time Delivery, OTD), los ciclos de pedido en los que no hubo faltantes (Cycle Service Level, CSL) y la proporción de piezas que se surten (Fill Rate, FR); además de los indicadores que en términos de unidades físicas, como las toneladas-kilometro o el nivel de facturación, son utilizados de forma general para medir la producción del autotransporte de carga (Blanquart \& Burmeister, 2009). Sin embargo, la elección de los indicadores para medir el desempeño de las operaciones logísticas depende de las necesidades de la empresa y, por lo tanto, estará en función de su operación y posicionamiento (Sánchez-Gómez, 2008).

De esta forma, sólo algunos de los indicadores citados resultan de gran utilidad para las empresas embarcadoras que contratan servicios de transporte, que tienen muchos proveedores, que embarcan carros completos (full truck load) y que, por el volumen de embarques, logran tener control en las negociaciones tarifarias. Por ejemplo, si la empresa embarcadora ha decidido focalizarse en el nivel de servicio y decide crear indicadores o criterios para la selección y la evaluación de sus proveedores de autotransporte -como la forma en que repartirá la facturación (viajes asignados)- se enfrenta a discrepancias como el tamaño de la empresa a contratar; es decir, la conveniencia entre las microempresas conformadas por choferes dueños de sus vehículos, conocidos como hombres-camión, o las empresas de mayor tamaño que tienen una estructura organizacional y mejores condiciones tecnológicas. Las diferencias se extienden a una 
serie de características que incluyen aspectos relacionado con los riesgos y exigencias, derivadas de la organización y control del trabajo, a las que se exponen los choferes durante la conducción.

De aquí surge la inquietud por saber qué variables relacionadas con las condiciones laborales, influyen en el desempeño de las empresas transportistas y sus incumplimientos en las entregas a tiempo. Para tratar de dar respuesta a estas interrogantes, se realiza este estudio de caso en una empresa de manufactura que domina cerca de $80 \%$ de las ventas totales en el mercado de sistemas de construcción ligera en México y, bajo la hipótesis de que las condiciones laborales y de salud influyen directamente en las entregas a tiempo, se recolectó información durante un periodo de dos años, con la que finalmente se construyó un modelo de regresión logística que permite calcular la probabilidad de incurrir en entregas tardías o incorrectas.

En total, se efectuaron 26,312 embarques y se llevó registro de las entregas fuera de tiempo, problemas de comunicación, siniestros y otros inconvenientes que, durante el tránsito, afectaron las entregas a tiempo (OTD) y, por lo tanto, repercutieron en el nivel de servicio logístico.

La empresa sólo carga camiones completos (full truck load) y utiliza tres tipos de vehículos: tráiler (77.37\%), torton (18.18\%) y vehículos con doble remolque, conocidos como full (4.45\%). Los embarques, fueron enviados desde tres plantas de producción, a 174 distribuidores, a los 32 estados que conforman la división política de México y tres ciudades de países de la frontera sur, con 1,697 choferes y 52 empresas de autotransporte, que fueron subcontratadas y trabajan divididas en tres zonas, que cubren cada una de las unidades productivas, a lo largo del país: norte, sur-centro y occidente-bajío.

\section{Revisión de la literatura}

\section{Las condiciones laborales de los choferes}

El tema de las condiciones laborales y de salud de los choferes ha sido ampliamente estudiado en la literatura médica internacional. Así, por ejemplo, existe una revisión crítica en la que se recopilaron 120 investigaciones sobre los riesgos para la salud y las tensiones de los camioneros, y en el que se clasifican en seis grupos sus principales clases de morbilidad: 1) Los trastornos psicológicos y psiquiátricos; 2) Perjuicios resultantes de los ciclos biológicos alterados; 3) Trastornos musculo-esqueléticos; 4) El cáncer y la morbilidad respiratoria; 5) Las enfermedades 
cardiovasculares; y 6) Los riesgos por el uso de sustancias y las prácticas sexuales. (Apostolopoulos, Sönmez, Shattell, \& Belzer, 2010).

En general, los estudios sobre condiciones laborales de los choferes indican que la actividad de conducir es una de las ocupaciones que generan mayores riesgos derivados de la ocupación (Bigert, 2004; Shin et al., 2013).

En primer lugar, se sabe que las enfermedades laborales y los accidentes de tránsito en los conductores del autotransporte de carga se relacionan con sus condiciones de trabajo; entre las que se incluyen el tiempo de conducción, las jornadas laborales o factores psicosociales, como el estrés o la satisfacción (Chun-Chieh et al., 2010; Van-Der-Beek, 2012). Además de que en comparación con otros grupos de conductores, los trabajadores del autotransporte tienen mayores tasas de mortalidad en los siniestros viales; y el riesgo de mortalidad se incrementa con el tamaño de los vehículos (Berrones, et al., 2018).

En segundo lugar, el estrés y la satisfacción en el trabajo de los conductores determinados por el confort, el nivel de ruido, la seguridad, los dispositivos tecnológicos y las características de los vehículos que utilizan (Ordaz et al., 2007)- tienen relación con la productividad y el desempeño de sus actividades (Bathija et al., 2014; Lámbarry, Trujillo, \& Cumbres, 2016). Estos factores psicosociales pueden ocasionar comportamientos negativos en el trabajo, por lo que resultan de gran importancia, no sólo por aumentar el riesgo de accidentes laborales sino por la cantidad de efectos adversos para la salud física y mental que se generan en este grupo de trabajo.

En cuanto a las condiciones laborales de los conductores en México, existe un estudio en el que se realizó una amplia búsqueda de investigaciones y en el que se identificaron cerca de 60 trabajos sobre conductores profesionales (Berrones, 2017). Aquí, se indica que la mayor cantidad de estudios dedicados a los conductores de transporte de carga, se enfocan en el uso de sustancias tóxicas, la fatiga, la somnolencia, el estrés laboral y los trastornos psicosomáticos, y que los choferes, además de ser susceptibles a problemas de salud, tienen la práctica común de desarrollar sus actividades en condiciones de precariedad laboral. 


\section{La regresión logística}

Los métodos de análisis multivariante de datos se han convertido en un componente esencial para estudiar variables de forma conjunta $\mathrm{y}$, desde los años setentas, estos métodos han probado su eficacia en el estudio de grandes cantidades de información (Pérez, 2004).

Actualmente, se debe seleccionar de una diversidad de técnicas, la más adecuada a las características de los datos y al objetivo de la investigación. Asimismo, además de tomar en cuenta los supuestos del análisis multivariante (normalidad, homocedasticidad, ausencia de multicolinealidad, entre otros), o la naturaleza de las variables (cualitativas o cuantitativas), se debe considerar la existencia de variables explicativas o explicadas. Así, Pérez (2004) clasifica las técnicas multivariantes en dos grandes grupos: 1) Los métodos de interdependencia, y 2) Los métodos de dependencia. Los primeros incluyen aquellos métodos donde ninguna variable destaca como dependiente principal, se utilizan para reducir la dimensión de un conjunto muy grande de datos o para clasificar a sus individuos en grupos más o menos homogéneos en relación con su perfil. Por su parte, los métodos de dependencia consideran que un conjunto de variables independientes explica una o varias variables dependientes a través de una ecuación o modelo que las relaciona y que, generalmente, permite predecir el valor de la variable dependiente.

Dentro de este último conjunto se encuentra el modelo de regresión logística binaria, que se utiliza para calcular la probabilidad de que ocurra o no un determinado suceso, conocido como variable dependiente y, cuya característica principal es la condición de ser cualitativa dicotómica (si o no; 1 ó 0). Además, la regresión logística tiene varias ventajas frente a otras técnicas que ayudan a predecir eventos o comportamientos futuros, tales como: las redes bayesianas, los árboles de decisión o las redes neuronales, entre otras. La regresión logística además de ser considerada una de las herramientas estadísticas más expresivas y versátiles para el análisis de datos en los fenómenos sociales (Fiuza \& Rodríguez, 2000), encuentra sus principales ventajas en:

1. Los cálculos de probabilidad y el modelo final son reducidos a una ecuación de tipo determinística, que simplifica los cálculos y las predicciones.

2. No sólo es ideal para la función explicativa de las variables, sino que permite, basada en una información reducida, explicarlas de forma descriptiva; ofreciendo, por ejemplo, 
datos referentes a la probabilidad de que ocurra el suceso de interés y la razón de momios (Odds de Ratio, OR).

3. Además de predecir riesgos, sirve para evaluar la magnitud de asociación entre los diferentes factores que se relacionan de manera independiente.

4. Utiliza variables categóricas de manera sencilla; ya que se crean variables ficticias (dummy) en las que se codifican las diferentes categorías de manera binaria.

5. No tiene la necesidad de crear algoritmos para las secuencias entre las variables, por lo que no requiere sistemas robustos.

\section{Aplicaciones de la regresión logística}

La regresión logística ha sido utilizada en múltiples aplicaciones relacionadas con las condiciones laborales, la cadena de suministro y en temas relacionados con la seguridad vial de los conductores. Así, por ejemplo, Al-Ghamdi (2002) utilizó la regresión logística para estimar la influencia de los factores de los accidentes de tránsito y para estimar su gravedad. Por su parte, otro estudio que tuvo una muestra de 684 conductores de camiones reclutados en la ciudad de Sao Paulo, y en el que se tuvo como objetivo estimar la prevalencia de accidentes, se propone un modelo de regresión logística para identificar los predictores de los siniestros (García et al., 2016). Por su parte, Nogueira et al. (2016) analizaron el abuso de alcohol y consumo frecuente según las características sociodemográficas de la población, para estimar el riesgo de accidente de tránsito; Hilakivi et al. (1989) utilizaron la regresión logística en la predicción de accidentes automovilísticos.

En cuanto a la cadena de suministro, las aplicaciones han sido muy diversas. Por nombrar unas cuantas, hay aplicaciones para calcular el riesgo crediticio en el financiamiento de la cadena de suministro en China (Zhu, Xie, Sun, Wang, \& Yan, 2016); para determinar la adopción de identificación por radiofrecuencia (Paydar, Endut, \& Lajevardi, 2013); para planificar la producción en la industria automotriz (Mehrjoo \& Bashiri, 2013) y para identificar los principales factores que impulsan a las compañías durante la selección de contratos en las cadenas de suministro de empresas europeas (Sluis \& Giovanni, 2016).

Por último, en cuanto a la salud ocupacional, la regresión logística se ha utilizado para evaluar el impacto de las condiciones laborales en la calidad de vida de los trabajadores del sector manufacturero en Colombia (Martínez, Oviedo, \& Luna, 2015); para investigar los factores 
ocupacionales asociados con la licencia por enfermedad en empleados australianos (Magee, Stefanic, Caputi, \& Iverson, 2011), para identificar los factores que determinan la satisfacción o insatisfacción en trabajadores españoles (Villar, Delgado, \& Barrilao, 2015), la duración de las horas de trabajo y su relación con la fatiga y el estrés laboral en trabajadores japoneses (Suwazono et al., 2008), y para determinar los indicadores de riesgo de incapacidad de trabajo por problemas de la espalda baja (Müller et al., 1999).

\section{El modelo de regresión logística}

Si $Y$, es una variable dependiente de tipo binomial y mutuamente excluyente, con probabilidad de ocurrencia $P(Y)$ que está influida por $K$ variables independientes $\left(X_{1}, X_{2}, \ldots, X_{k}\right)$. Y, dado que la razón de probabilidad de éxito, conocida como odds, es igual a la probabilidad de que ocurra un evento $P(Y)$ entre la probabilidad de que no ocurra 1-P(Y). Entonces, si se utiliza el logaritmo natural $(\ln )$ para normalizar la probabilidad de la variable dicotómica, y se forma la ecuación básica de la regresión logística:

$$
\ln [P(Y) /(1-P(Y))]=\beta_{0}+\beta_{1} X_{1}+\beta_{2} X_{2}+\cdots+\beta_{k} X_{k}
$$

Donde $\beta$ simboliza a los $K$ coeficientes de regresión que, sin significar causalidad, representan el cambio de la variable dependiente por unidad en las $K$ variables independientes.

De acuerdo con Álvarez-Cáceres (2007), el método más utilizado para calcular los coeficientes de regresión logística, es a través de la función de verosimilitud (likelihood function):

$$
\operatorname{Lo}(\beta)=P(Y)^{\sum_{j=1}^{n} y_{j}} \cdot(1-p(y))^{\left(n-\sum_{j=1}^{n} y_{i}\right)}
$$

Y el logaritmo neperiano de la función de verosimilitud:

$$
\operatorname{LLo}(\beta)=\sum_{j=1}^{n} y_{j} \cdot \ln (P(Y))+\left(n-\sum_{j=1}^{n} y_{j}\right) \cdot \ln (1-p(Y))
$$

Donde $\beta$ es el vector de coeficientes de regresión logística $\left(\beta_{1}, \beta_{2}, \ldots, \beta_{k}\right)$ y la $y_{j}$ representa los jésimos valores, que toma valores de 1 y 0 , en la variable dependiente $Y$. 
Así, una vez estimados los coeficientes de regresión logística, para calcular la probabilidad de ocurrencia, se puede despejar $P(Y)$ de la ecuación básica de la regresión logística:

$$
P(Y)=\frac{1}{1+e^{-\left(\beta_{0}+\beta_{1} X_{1}\right)}}
$$

Finalmente, para terminar este breve resumen del modelo de regresión logística, se debe recordar, que cuando las variables independientes, son cualitativas u ordinales, se tienen que añadir tantas variables ficticias (dummy), como categorías tiene la variable que se introduce. Y, además de verificar la validez y ajuste del modelo con pruebas como el logaritmo de la verosimilitud -2, o las pruebas de Hosmer y Lemeshow, se debe buscar su forma parsimónica, comprobando la significancia de cada uno de los coeficientes -mediante la prueba de $t$ de Student o de Wald- para tener un modelo de regresión logística, en el que todas las variables que se incluyen son significativas, tanto del punto de vista técnico como estadístico.

\section{Método}

Se analizó la información de 26,312 embarques realizados durante dos años en una empresa que manufactura sistemas de construcción ligera en México. Variables como la relación laboral (empleado u hombre-camión), el acceso a la seguridad social, la edad y los resultados de una evaluación subjetiva del estrés y la satisfacción de los conductores del autotransporte de carga, fueron utilizadas para determinar los factores que afectan el desempeño en las entregas a tiempo. En total, se consideraron inicialmente once variables independientes, mismas que se describen en la tabla 1.

Después de examinar las características de la distribución de las variables implicadas en el análisis, las relaciones bivariantes y multivariantes, y el análisis de las diferencias entre grupos es decir, de analizar las variables individuales y las relaciones entre ellas-, los datos se probaron en varios modelos de tipo multivariante. Finalmente, dado que la variable dependiente, a la que se le nombró incumplimiento de transporte (Inc_Tpe), tiene la característica de ser dicotómica (entrega correcta o entrega incorrecta) se encontró adecuado un enfoque de regresión logística. De esta forma, para examinar la contribución de las variables independientes (tabla 1) se aplicó una regresión logística a los datos relacionados con las entregas de los embarques, se verificó su significancia y se construyó un modelo de tipo parsimónico. 
Entregas tardías o incorrectas en el autotransporte de carga y su relación con las condiciones laborales de los choferes: Un modelo de regresión logística

Tabla 1. Definición y categorización de variables.

\begin{tabular}{|c|c|c|c|c|}
\hline Variable & Clave & Descripción & Valores & $\begin{array}{l}\text { Tipo de } \\
\text { variable }\end{array}$ \\
\hline $\begin{array}{l}\text { Tipo de } \\
\text { Vehículo }\end{array}$ & $T V$ & $\begin{array}{l}\text { Se refiere a los vehículos que se utilizaron para } \\
\text { realizar la carga de los embarques. Categorizados de } \\
\text { acuerdo a la NOM-012-SCT-2-2014. }\end{array}$ & $\begin{array}{l}\text { 1. C3 (Torton) } \\
\text { 2. T3R3 (Tráiler) } \\
\text { 3. T3S2R4 (Full) }\end{array}$ & $\begin{array}{l}\text { Cualitativa } \\
\text { nominal }\end{array}$ \\
\hline $\begin{array}{l}\text { Acceso a la } \\
\text { Seguridad } \\
\text { Social }\end{array}$ & $S S$ & $\begin{array}{l}\text { Indica si los conductores tienen acceso a las } \\
\text { prestaciones laborales que exige la normatividad en } \\
\text { materia de trabajo, para asegurar la asistencia médica y } \\
\text { el ingreso en caso de vejez, enfermedad, invalidez y } \\
\text { accidentes de trabajo, entre otras causas. }\end{array}$ & $\begin{array}{l}\text { 0. Sin acceso } \\
\text { 1. Con acceso }\end{array}$ & $\begin{array}{l}\text { Cualitativa } \\
\text { nominal }\end{array}$ \\
\hline $\begin{array}{l}\text { Tiempo de } \\
\text { Tránsito }\end{array}$ & $T T$ & $\begin{array}{l}\text { Es el tiempo programado de viaje (en horas) que } \\
\text { tienen que realizar los conductores desde el punto de } \\
\text { embarque hasta el destino final. }\end{array}$ & Tiempo en horas & $\begin{array}{l}\text { Cuantitativ } \\
\text { a continua }\end{array}$ \\
\hline $\begin{array}{l}\text { Frecuencia de } \\
\text { Estrés }\end{array}$ & Est & $\begin{array}{l}\text { Es la frecuencia percibida del estado de cansancio } \\
\text { mental que los conductores declararon en cada viaje. } \\
\text { Se determinó de forma subjetiva, con opciones de tipo } \\
\text { Likert, en respuesta a la pregunta ¿Con qué frecuencia } \\
\text { sientes estrés en el trabajo? }\end{array}$ & $\begin{array}{l}\text { 1. Nunca } \\
\text { 2. Casi nunca } \\
\text { 3. Algunas veces } \\
\text { 4. Frecuentemente } \\
\text { 5. Siempre }\end{array}$ & $\begin{array}{l}\text { Cualitativa } \\
\text { ordinal }\end{array}$ \\
\hline $\begin{array}{l}\text { Nivel de } \\
\text { Insatisfacción }\end{array}$ & Ins & $\begin{array}{l}\text { Es el sentimiento de inquietud, de frustración o de falta } \\
\text { del cumplimiento de las expectativas, que de manera } \\
\text { subjetiva los conductores evaluaron con una escala de } \\
\text { tipo Likert. }\end{array}$ & $\begin{array}{l}\text { 1. Muy satisfecho } \\
\text { 2. Satisfecho } \\
\text { 3. Ni satisfecho, ni } \\
\text { insatisfecho } \\
\text { 4. Insatisfecho } \\
\text { 5. Muy insatisfecho }\end{array}$ & $\begin{array}{l}\text { Cualitativa } \\
\text { ordinal }\end{array}$ \\
\hline $\begin{array}{l}\text { Zona } \\
\text { Trabajo }\end{array}$ & $Z T$ & $\begin{array}{l}\text { Se refiere al espacio geográfico del país, dividido en } \\
\text { tres partes, donde usualmente trabajan los conductores. }\end{array}$ & $\begin{array}{l}\text { 1. Norte } \\
\text { 2. Sur Centro } \\
\text { 3. Occidente Bajío }\end{array}$ & $\begin{array}{l}\text { Cualitativa } \\
\text { nominal }\end{array}$ \\
\hline Edad & $E d a$ & $\begin{array}{l}\text { Tiempo (en años) que ha vivido el conductor hasta el } \\
\text { momento de realizar el viaje. }\end{array}$ & $18-99$ & $\begin{array}{l}\text { Cuantitativ } \\
\text { a continua }\end{array}$ \\
\hline Enfermedad & Enf & $\begin{array}{l}\text { De manera subjetiva, los trabajadores declararon tener } \\
\text { o no alguna alteración en su salud. }\end{array}$ & $\begin{array}{l}\text { 0. No está enfermo } \\
\text { 1. Si está enfermo }\end{array}$ & $\begin{array}{l}\text { Cualitativa } \\
\text { nominal }\end{array}$ \\
\hline $\begin{array}{l}\text { Propiedad del } \\
\text { Vehículo }\end{array}$ & $P V$ & $\begin{array}{l}\text { Los conductores declararon su estado, en relación a la } \\
\text { propiedad de los vehículos. En este campo se distingue } \\
\text { entre los que son trabajadores autónomos y los que } \\
\text { venden su fuerza de trabajo. }\end{array}$ & $\begin{array}{l}0 . \text { No es dueño del } \\
\text { vehículo } \\
\text { 1. Si es dueño del } \\
\text { vehículo }\end{array}$ & $\begin{array}{l}\text { Cualitativa } \\
\text { nominal }\end{array}$ \\
\hline $\begin{array}{l}\text { Tamaño de } \\
\text { empresa }\end{array}$ & $T E$ & $\begin{array}{l}\text { Se indica el tipo de empresa de autotransporte en la } \\
\text { que los conductores desarrollan sus actividades } \\
\text { laborales; categorizadas por el número de vehículos de } \\
\text { acuerdo a la clasificación oficial en México (DGAF, } \\
\text { 2015). }\end{array}$ & $\begin{array}{l}\text { 1. Hombre-Camión (1- } \\
\text { 5) } \\
\text { 2. Pequeña }(6-30) \\
\text { 3. Mediana }(31-100) \\
\text { 4. Grande }(100+)\end{array}$ & $\begin{array}{l}\text { Cualitativa } \\
\text { ordinal }\end{array}$ \\
\hline $\begin{array}{l}\text { Satisfacción } \\
\text { con el ingreso }\end{array}$ & $S I$ & $\begin{array}{l}\text { Es el sentimiento de bienestar o placer en relación al } \\
\text { dinero que perciben los conductores por su trabajo y } \\
\text { que, de manera subjetiva, declararon en una escala de } \\
\text { tipo Likert. }\end{array}$ & $\begin{array}{l}\text { 1. Muy insatisfactorio } \\
\text { 2. Insatisfactorio } \\
\text { 3. Ni satisfactorio, ni } \\
\text { insatisfactorio } \\
\text { 4. Satisfactorio } \\
\text { 5. Muy satisfactorio }\end{array}$ & $\begin{array}{l}\text { Cualitativa } \\
\text { ordinal }\end{array}$ \\
\hline
\end{tabular}

No 20, Vol. 10 (1), 2018. ISSN 2007 - 0705, pp.: 413 - 441 


\begin{tabular}{|c|c|c|c|c|}
\hline $\begin{array}{l}\text { Incumplimient } \\
\text { o de Entrega }\end{array}$ & $\begin{array}{l}\text { Inc_Tp } \\
e\end{array}$ & $\begin{array}{l}\text { Se refiere a los embarques evaluados de manera } \\
\text { negativa, con causas atribuidas al transporte, tales } \\
\text { como daños en las mercancías, demoras en el tiempo } \\
\text { de tránsito o cualquier tipo de inconformidad en el } \\
\text { destino. }\end{array}$ & $\begin{array}{l}\text { 0. Entrega correcta } \\
\text { 1. Entrega Incorrecta }\end{array}$ & $\begin{array}{l}\text { Cualitativa } \\
\text { nominal }\end{array}$ \\
\hline
\end{tabular}

Fuente: Elaboración propia.

\section{La empresa de embarcadora}

La empresa, que fabrica aproximadamente $80 \%$ del total de los sistemas de construcción ligera que se comercializan en México, realiza sus embarques en vehículos pesados que son cargados en tres plantas, ubicadas en el norte, oriente y occidente del país. Las diferentes localizaciones fabrican los mismos productos, con los mismos estándares de calidad, y son embarcados, para distribuirse en todo el territorio nacional bajo el mismo proceso en tres tipos de vehículos: camiones unitarios tipo torton (C3), camiones articulados tipo tráiler (T3S3) y doblemente articulados conocidos como full (T3S2R4), con capacidad de 19, 30 y 56 toneladas de carga útil, respectivamente.

\section{Las empresas de autotransporte}

La empresa de manufactura no tiene flota vehicular propia, por lo que todo el transporte de las mercancías se realiza como una actividad subcontratada. En total, se utilizaron 52 empresas de autotransporte que fueron clasificadas por el número y tipo de vehículos disponibles (tamaño de empresa), la planta o zona de trabajo en la que prestan el servicio, norte, sur-centro y occidentebajío, el acceso a la seguridad social y la propiedad del vehículo, es decir, si el chofer es dueño o no.

\section{Sistematización de los datos}

La información que se utiliza en este estudio fue obtenida a través del registro sistemático de datos, en un módulo nombrado Sistema de Embarques, programado y adaptado, como complemento de un sistema de Planeación de Recursos Empresariales (ERP), y que tiene como principal objetivo crear indicadores para los servicios de transporte que la empresa embarcadora subcontrata. Los datos se dividen en tres componentes: seguimiento al embarque, información de entrega, y percepción del chofer.

El seguimiento al embarque registra el horario en que el chofer arriba al punto de carga y se encuentra disponible para cargar, la hora en que se posiciona en el andén, la hora de inicio y 
término del proceso de carga y el horario de salida en el que se inició el viaje al destino final. En todos estos procesos, varios usuarios utilizan diferentes ordenadores, registran las fechas seleccionando una casilla de verificación, que no puede ser manipulada por los usuarios y donde los horarios son capturados en tiempo real. Estos datos, son comparados, con los horarios planeados y se verifica el cumplimiento de los transportistas y del personal del área de carga.

La información de entrega, se verifica a través de sistemas de posicionamiento global, si el transportista dispone del servicio, o de reportes telefónicos que realizan los choferes; posteriormente, son verificados a través de la documentación (notas de remisión), en la que el cliente firma de recibido, reporta faltantes o daños aparentes a los materiales y establece la fecha y hora en la que arribó el transportista; misma que se contrasta con la fecha en la que se prometió la entrega.

Finalmente, para evaluar la percepción del chofer, en los documentos se incluyeron tres preguntas obligatorias de tipo Likert, con escala del uno al cinco, que evaluaron el nivel de estrés laboral, la insatisfacción en el trabajo y la satisfacción con el ingreso. Otros datos, como el nombre, la edad del chofer y si cuenta con seguridad social, fueron introducidos previamente en el registro de conductores y durante la planeación de los embarques.

Dado que se identificó que los embarques entregados se califican de manera dicotómica, se utilizó como variable dependiente y se codificó con un uno $(Y=1)$ a los embarques que fueron entregados de forma incorrecta, es decir, con daños, fuera de tiempo, o cualquier causa de incumplimiento; y con un cero $(Y=0)$ para los embarques que se entregaron en tiempo y forma. Esta clasificación, incluye sólo los embarques cuyas causas de incumplimiento fueron atribuidas a los transportistas, y se excluyen los derivados por cualquier otra causa, como errores de programación o problemas en las zonas de carga.

Se utilizó el software SPSS para evaluar los coeficientes. Aunque existen otros programas estadísticos de mayor potencia, que generan la regresión logística y que proveen medidas de asociación entre las variables de respuesta y probabilidades predichas, el software SPSS fue seleccionado debido a que permite la recodificación de variables y registros según las necesidades del usuario; lo que simplifica el manejo de gran cantidad de información, facilita la limpieza y permite ordenar los datos de manera muy eficaz. Aunado a esto, el software SPSS se adecuó a las pruebas requeridas, como es el caso de la prueba paramétrica de Wald; ya que, por ejemplo, aunque el SPSS y Minitab generan los mismos valores de los parámetros estimados, los 
límites del intervalo de confianza, ambos al 95\%, son diferentes. Esto debido a que el SPSS usa los valores chi-cuadrado de Wald, mientras que Minitab utiliza la aproximación de la distribución normal estándar.

De esta forma, a través de la prueba de Ómnibus y mediante el valor del incremento del estadístico $-2 \log$, se rechazó la hipótesis nula $\left(H_{\mathrm{o}}: \beta_{1}=\beta_{2}=\ldots \beta_{\mathrm{i}}=0\right)$ del contraste general del modelo, que indica que al menos uno de los coeficientes de las variables es distinto de cero. Posteriormente, se comprobó, mediante las pruebas de la t de Student y de Wald, si los

coeficientes de regresión logística eran significativos. Se eliminaron las variables que no cumplieron la condición, se ajustó el modelo con las variables cuyos coeficientes fueron estadísticamente significativos, y se volvió a ejecutar el modelo.

Asimismo, se realizó la bondad del ajuste para contrastar si el modelo se ajustaba a los datos; este proceso es conocido como la prueba de Hosmer-Lemeshow y consiste en comprobar la bondad de ajuste de los datos, a una distribución teórica chi-cuadrado, en la que se crea una tabla para comparar los valores observados y los esperados, tanto de si ocurre la entrega incorrecta de los embarques, como la correcta.

Finalmente, se utilizaron los coeficientes de regresión logística para elaborar una ecuación que permite calcular la probabilidad de incumplimiento en las entregas a tiempo de los choferes del autotransporte de carga. De esta forma, se identificaron las categorías o valores que toman las variables y que generan tanto la menor, como la mayor probabilidad de incumplimiento y que, por lo tanto, se pueden considerar consideran factores protectores o factores de riesgos para los cumplimientos en las entregas a tiempo.

\section{Resultados}

De los 26,312 embarques, casi 14\% $(3,635)$ fueron registrados como entregas incorrectas que incluían cualquier causa y no sólo cuestiones de transporte; es decir, se entregaron con algún faltante, con materiales equivocados, con daños o no se cumplió con el horario de entrega prometido. De los incumplimientos, 1,790 (6.81\%) fueron atribuidos a causas relacionadas con los transportistas; representan el suceso de interés y se codificaron con $Y=1$. El resto de los embarques fueron considerados entregas correctas y fueron codificados con $Y=0$.

Los porcentajes de los embarques correctos e incorrectos, clasificados por algunas variables relacionadas a la organización del trabajo y la prueba chi-cuadrado para probar su independencia, 
Entregas tardías o incorrectas en el autotransporte de carga y su relación con las condiciones laborales de los choferes: Un modelo de regresión logística

se encuentran en la tabla 2. Se puede observar, que las entregas correctas de los embarques presentan dependencia de las variables relacionadas al tamaño de empresa, la zona de trabajo y el tipo de vehículo; asimismo, los mayores porcentajes de entregas incorrectas se presentan en las empresas pequeñas (2.6\%), en la zona sur-centro (3.9\%) y en el vehículo de tipo tráiler (5.1\%).

Tabla 2. Embarques correctos e incorrectos según variables de tamaño de empresa, vehículos y zona de trabajo.

\begin{tabular}{|c|c|c|c|c|c|c|c|}
\hline \multirow{2}{*}{ Variable } & \multicolumn{2}{|c|}{ Correctos $Y=0$} & \multicolumn{2}{|c|}{ Incorrectos $Y=1$} & \multirow{2}{*}{$\begin{array}{l}\text { Total } \\
\text { Número }\end{array}$} & \multirow[b]{2}{*}{$(\%)^{a}$} & \multirow{2}{*}{$\begin{array}{l}P \text {-value } \\
\chi^{2} \text {-Test }\end{array}$} \\
\hline & Número & $(\%)^{\mathrm{a}}$ & Número & $(\%)^{\mathrm{a}}$ & & & \\
\hline \multicolumn{8}{|l|}{ Tamaño de empresa } \\
\hline \multicolumn{8}{|l|}{ Hombre-Camión (1- } \\
\hline 5) & 6,838 & $(26.0 \%)$ & 638 & $(2.4 \%)$ & 7,476 & $(28.4 \%)$ & 0.000 \\
\hline Pequeña (6-30) & 9,222 & $(35.0 \%)$ & 678 & $(2.6 \%)$ & 9,900 & $(37.6 \%)$ & \\
\hline Mediana (31-100) & 5,918 & $(22.5 \%)$ & 313 & $(1.2 \%)$ & 6,231 & $(23.7 \%)$ & \\
\hline Grande (100+) & 2,542 & $(9.7 \%)$ & 163 & $(0.6 \%)$ & 2,705 & $(10.3 \%)$ & \\
\hline \multicolumn{8}{|l|}{ Zona de trabajo } \\
\hline Norte & 10,022 & $(38.1 \%)$ & 613 & $(2.3 \%)$ & 10,635 & $(40.4 \%)$ & 0.000 \\
\hline Sur-Centro & 11,532 & $(43.8 \%)$ & 1,018 & $(3.9 \%)$ & 12,550 & $(47.7 \%)$ & \\
\hline Occidente-Bajío & 2,966 & $(11.3 \%)$ & 161 & $(0.6 \%)$ & 3,127 & $(11.9 \%)$ & \\
\hline \multicolumn{8}{|l|}{ Tipo de vehículo } \\
\hline C3 (Torton) & 4,448 & $(16.9 \%)$ & 335 & $(1.3 \%)$ & 4,783 & $(18.2 \%)$ & 0.028 \\
\hline T3R3 (Tráiler) & 19,002 & $(72.2 \%)$ & 1,355 & $(5.1 \%)$ & 20,357 & $(77.4 \%)$ & \\
\hline T3S2R4 (Full) & 1,070 & $(4.1 \%)$ & 102 & $(0.4 \%)$ & 1,172 & $(4.5 \%)$ & \\
\hline Total & 24,520 & $(93.2 \%)$ & 1,792 & $(6.8 \%)$ & 26,312 & $(100 \%)$ & \\
\hline
\end{tabular}

Respecto a los conductores, poco más de una cuarta parte (25.5\%) son dueños de sus vehículos (hombre-camión), una quinta parte $(20.6 \%)$ no tienen prestaciones sociales, en promedio tienen una edad de 40.7 \pm 12.99 años, y $8.4 \%$ indicaron tener alguna enfermedad permanente; principalmente de tipo cardiovascular o crónica degenerativa. Además, bajo una escala del uno al cinco (cuyos valores se pueden observar en la tabla 1), se obtuvo un promedio de 2.6 para la satisfacción con el ingreso, 2.4 para el estrés y 2.4 para la insatisfacción en su trabajo.

En la tabla 3 (valores promedio por tipo de empresa) se puede observar que, para las variables subjetivas evaluadas con escalas de tipo Likert, los conductores cuyos embarques 
fueron calificados como incorrectos valoran de forma más negativa el estrés (4.3) y la insatisfacción laboral (3.5), en comparación con los que realizaron embarques correctos (2.2 para estrés y 2.3 para insatisfacción); no así, para la satisfacción con el ingreso, dónde los conductores con embarques correctos e incorrectos, promedian la misma evaluación (2.6).

Tabla 3. Valores promedios por tipo de empresa y tipo de entrega.

\begin{tabular}{|c|c|c|c|c|c|c|}
\hline \multirow{2}{*}{\multicolumn{2}{|c|}{ Variable/Entrega }} & \multicolumn{5}{|c|}{ Tipo de empresa } \\
\hline & & \multirow[t]{2}{*}{$\begin{array}{l}\text { Hombre- } \\
\text { Camión }\end{array}$} & \multirow[t]{2}{*}{ Pequeña } & \multirow[t]{2}{*}{ Mediana } & \multirow[t]{2}{*}{ Grande } & \multirow[t]{2}{*}{ Total } \\
\hline Edad (a & & & & & & \\
\hline & Correcto & 41.4 & 41.0 & 39.9 & 40.1 & 40.7 \\
\hline & Incorrecto & 41.1 & 41.4 & 39.3 & 39.4 & 40.8 \\
\hline & Total & 41.4 & 41.0 & 39.8 & 40.0 & 40.7 \\
\hline \multicolumn{7}{|l|}{ Estrés } \\
\hline & Correcto & 2.2 & 2.3 & 2.2 & 2.2 & 2.2 \\
\hline & Incorrecto & 4.3 & 4.3 & 4.3 & 4.3 & 4.3 \\
\hline & Total & 2.4 & 2.4 & 2.3 & 2.4 & 2.4 \\
\hline \multicolumn{7}{|c|}{ Insatisfacción } \\
\hline & Correcto & 2.3 & 2.4 & 2.3 & 2.3 & 2.3 \\
\hline & Incorrecto & 3.6 & 3.5 & 3.5 & 3.5 & 3.5 \\
\hline & Total & 2.4 & 2.4 & 2.4 & 2.4 & 2.4 \\
\hline \multicolumn{7}{|l|}{ Ingreso } \\
\hline & Correcto & 2.7 & 2.5 & 2.7 & 2.4 & 2.6 \\
\hline & Incorrecto & 2.7 & 2.6 & 2.8 & 2.3 & 2.6 \\
\hline & Total & 2.7 & 2.6 & 2.7 & 2.4 & 2.6 \\
\hline
\end{tabular}

Fuente: Elaboración propia.

\section{Entregas incorrectas y su relación con las condiciones laborales: El modelo de regresión logística binaria}

Utilizando el software estadístico SPSS, se ejecutó dos veces el modelo de regresión logística; la primera con todas las variables, y la segunda sólo con las variables que resultaron significativas a un nivel de confianza con $\alpha=0.05$. Aquí, se puede precisar que los estimadores por máxima verosimilitud de los parámetros del modelo son asintóticamente normales y su matriz de varianzas es perfectamente calculable a partir del algoritmo de la función de máxima verosimilitud.

De esta forma, un intervalo de confianza al (1- $\alpha) \%$ para el estimador del coeficiente $\alpha_{\mathrm{i}}$, se calcula con la expresión: $\widehat{\alpha}_{\iota} \pm Z_{\alpha / 2} \hat{\sigma}\left(\hat{\alpha}_{i}\right)$; y el estadístico del contraste para los coeficientes está dado por la hipótesis nula $\left(\mathrm{H}_{\mathrm{o}}: \alpha_{\mathrm{i}}=0\right)$ que implica que la variable dependiente $(\mathrm{Y})$ no depende de la $\mathrm{X}_{\mathrm{i}}$; y, por lo tanto, esta última se debe excluir del modelo (Pérez, 2004). Asimismo, la región 
Entregas tardías o incorrectas en el autotransporte de carga y su relación con las condiciones laborales de los choferes: Un modelo de regresión logística

crítica estará dada por $|\mathrm{Z}|>\mathrm{Z}_{\alpha / 2}$, donde $\mathrm{Z}=\left(\widehat{\alpha}_{\imath}-a\right) /\left[\hat{\sigma}\left(\hat{\alpha}_{i}\right)\right] \rightarrow N\left(a, \hat{\sigma}\left(\hat{\alpha}_{i}\right)\right)$ o por el contraste estadístico de Wald, definido como $\mathrm{W}=\mathrm{Z}^{2}$ y cuya distribución es una chi-cuadrado con un grado de libertad, que tiene su región critica de contraste en $\mathrm{W}>\chi^{2}{ }_{\alpha}$.

Asimismo, el software estima los parámetros del modelo, los intervalos y los contrastes de hipótesis sobre los coeficientes; por lo que sistematiza los cálculos y reduce el procedimiento a la verificación del contraste de hipótesis -aunque SPSS también lo hace de forma automática- y, así, se verifica si la probabilidad que mide la evidencia en contra de la hipótesis nula (valor $p$ ) indica asociación estadísticamente significativa $(\mathrm{p}<\alpha)$ o si la variable debe ser excluida del modelo $(\mathrm{p}>\alpha)$, por no ser estadísticamente significativa.

De esta forma, en la primera ejecución en la que se incluyeron todas las variables, resultaron no ser significativas (p>.05) las variables $Z T, E d a, E n f, P V, T E$, y el $D I$, por lo que fueron excluidas y, en la siguiente ejecución, se incluyeron sólo las variables restantes $T V$, Est, Ins, SS y TT; mismas que no demostraron evidencia de independencia y fueron incluidas en el modelo final.

Los coeficientes estimados, la prueba de Wald, los valores de odds de ratio $\left(\mathrm{OR}=e^{\beta}\right)$ y los intervalos de confianza para la muestra de estimación se presentan en la tabla 4.

Tabla 4. Variables en la ecuación.

\begin{tabular}{|c|c|c|c|c|c|c|c|c|c|}
\hline \multirow[t]{2}{*}{ Ejecución } & \multirow[t]{2}{*}{ Variable } & \multirow[t]{2}{*}{ B } & \multirow{2}{*}{$\begin{array}{l}\text { Error } \\
\text { estándar }\end{array}$} & \multirow[t]{2}{*}{ Wald } & \multirow[t]{2}{*}{ gl } & \multirow[t]{2}{*}{ Sig. } & \multirow[t]{2}{*}{$\operatorname{Exp}(B)$} & \multicolumn{2}{|c|}{$\begin{array}{c}\text { 95\% C.I. para } \\
\operatorname{EXP}(B)\end{array}$} \\
\hline & & & & & & & & Inferior & Superior \\
\hline \multirow[t]{14}{*}{1} & Eda & 0.001 & 0.003 & 0.117 & 1 & .733 & 1.001 & .995 & 1.007 \\
\hline & $\operatorname{Enf}(1)$ & 0.137 & 0.126 & 1.186 & 1 & .276 & 1.147 & .896 & 1.468 \\
\hline & $\mathrm{PV}(1)$ & 0.007 & 0.103 & 0.005 & 1 & .944 & 1.007 & .823 & 1.232 \\
\hline & $\operatorname{SI}(5)$ & & & 1.986 & 4 & .738 & & & \\
\hline & $\mathrm{SI}(1)$ & -0.189 & 0.141 & 1.800 & 1 & .180 & .828 & .629 & 1.091 \\
\hline & $\mathrm{SI}(2)$ & -0.124 & 0.141 & 0.772 & 1 & .379 & .884 & .670 & 1.165 \\
\hline & SI(3) & -0.160 & 0.132 & 1.464 & 1 & .226 & .852 & .657 & 1.104 \\
\hline & $\mathrm{SI}(4)$ & -0.130 & 0.159 & 0.667 & 1 & .414 & .878 & .644 & 1.199 \\
\hline & $\mathrm{TE}(4)$ & & & 5.454 & 3 & .141 & & & \\
\hline & $\mathrm{TE}(3)$ & -0.339 & 0.188 & 3.250 & 1 & .071 & .713 & .493 & 1.030 \\
\hline & $\mathrm{TE}(2)$ & -0.350 & 0.150 & 5.402 & 1 & .020 & .705 & .525 & .947 \\
\hline & $\mathrm{TE}(1)$ & -0.212 & 0.154 & 1.891 & 1 & .169 & .809 & .598 & 1.094 \\
\hline & $\mathrm{ZT}(3)$ & & & 1.827 & 2 & .401 & & & \\
\hline & $\mathrm{ZT}(1)$ & -0.116 & 0.177 & 0.428 & 1 & .513 & .891 & .630 & 1.260 \\
\hline
\end{tabular}


Berrones Sanz, Luis David et al.

\begin{tabular}{|c|c|c|c|c|c|c|c|c|c|}
\hline & $\mathrm{ZT}(2)$ & 0.082 & 0.136 & 0.364 & 1 & .546 & 1.085 & .832 & 1.416 \\
\hline \multirow[t]{16}{*}{2} & Est(5) & & & $2,810.418$ & 4 & .000 & & & \\
\hline & Est(1) & -5.738 & 0.238 & 581.059 & 1 & .000 & .003 & .002 & .005 \\
\hline & Est(2) & -5.693 & 0.138 & $1,690.616$ & 1 & .000 & .003 & .003 & .004 \\
\hline & Est(3) & -4.299 & 0.115 & $1,389.720$ & 1 & .000 & .014 & .011 & .017 \\
\hline & Est(4) & -0.853 & 0.089 & 91.856 & 1 & .000 & .426 & .358 & .507 \\
\hline & $\operatorname{Ins}(5)$ & & & $1,078.105$ & 4 & .000 & & & \\
\hline & $\operatorname{Ins}(1)$ & -2.300 & 0.137 & 281.146 & 1 & .000 & .100 & .077 & .131 \\
\hline & $\operatorname{Ins}(2)$ & -3.741 & 0.128 & 848.747 & 1 & .000 & .024 & .018 & .031 \\
\hline & $\operatorname{Ins}(3)$ & -2.050 & 0.120 & 291.503 & 1 & .000 & .129 & .102 & .163 \\
\hline & $\operatorname{Ins}(4)$ & -0.569 & 0.129 & 19.550 & 1 & .000 & .566 & .440 & .729 \\
\hline & $\mathrm{SS}(1)$ & -0.578 & 0.090 & 40.899 & 1 & .000 & .561 & .470 & .670 \\
\hline & $\mathrm{TT}$ & -0.007 & 0.003 & 6.334 & 1 & .012 & .993 & .988 & .999 \\
\hline & $\mathrm{TV}(3)$ & & & 31.527 & 2 & .000 & & & \\
\hline & $\mathrm{TV}(1)$ & -1.188 & 0.214 & 30.789 & 1 & .000 & .305 & .200 & .464 \\
\hline & $\mathrm{TV}(2)$ & -0.751 & 0.179 & 17.506 & 1 & .000 & .472 & .332 & .671 \\
\hline & Constante & 3.792 & 0.239 & 252.310 & 1 & .000 & 44.356 & & \\
\hline
\end{tabular}

$\mathbf{B}=$ Coeficiente de regresión. $\mathbf{g l}=$ Grados de libertad.

Sig.=Significancia. Wald: Prueba estadística paramétrica que se distribuye según una $\chi^{2} \cdot \operatorname{Exp}(\mathbf{B}):$ Odds ratio correspondiente al aumento de una unidad en el valor de la variable. C.I. = Intervalo de confianza. Fuente:

Elaboración propia (Resultados en SPSS).

Con los coeficientes de regresión $\beta_{\mathrm{i}}$, que resultaron significativos, se forma el modelo para calcular la probabilidad de incumplimiento del transporte (Inc_Tpe), mismo que queda de la siguiente forma:

$$
P\left(I n c_{-} T p e\right)=\frac{1}{1+e^{-Z}}
$$

Donde:

$$
\begin{array}{r}
Z=B_{I n s(j)} X_{I n s j)}+B_{E s t(j)} X_{E s t(j)}+B_{T V(j)} X_{T V(j)}-0.578 * X_{S S(j)}-0.007 * T T+3.792 \\
X_{i j}=\left\{\begin{array}{l}
0 ; \text { Si no pertenece a la categoría. } \quad i=T V, E s t, \text { Ins, SS } \\
1 ; \text { Si pertenece a la categoría. } \quad ;=1,2,3, \ldots, n
\end{array}\right.
\end{array}
$$

Y:

$$
\text { TT = Tiempo de Tránsito en horas. }
$$


Entregas tardías o incorrectas en el autotransporte de carga y su relación con las condiciones laborales de los choferes: Un modelo de regresión logística

Durante la ejecución, se crearon variables ficticias (variables dummy) que representan las categorías de cada variable; ya que las variables cualitativas no pueden ser incluidas directamente en el modelo y se tienen que crear tantas variables dummy, como categorías tenga la variable. Estas variables son dicotómicas, y tienen el valor 1 si pertenece a la categoría y 0 cuando no pertenece. Las codificaciones de las variables se muestran en la siguiente tabla 5.

Tabla 5. Codificaciones de variables categóricas.

\begin{tabular}{|c|c|c|c|c|c|c|c|}
\hline \multirow{2}{*}{$\begin{array}{l}\text { Variable } \\
\text { (i) }\end{array}$} & \multirow{2}{*}{$\begin{array}{l}\text { Valor } \\
\text { (j) }\end{array}$} & \multirow{2}{*}{$\begin{array}{l}\text { Clave } \\
\left(\mathbf{X}_{\mathrm{ij}}\right)\end{array}$} & \multirow{2}{*}{ Frecuencia } & \multicolumn{4}{|c|}{ Codificación de parámetro } \\
\hline & & & & (1) & (2) & (3) & (4) \\
\hline \multirow{5}{*}{$\begin{array}{l}\text { Insatisfacción } \\
\text { (Ins) }\end{array}$} & 1. Muy satisfecho & $\operatorname{Ins}(1)$ & 3,286 & 1.000 & 0.000 & 0.000 & 0.000 \\
\hline & 2. Satisfecho & $\operatorname{Ins}(2)$ & 13,613 & 0.000 & 1.000 & 0.000 & 0.000 \\
\hline & $\begin{array}{l}\text { 3. Ni satisfecho, ni } \\
\text { insatisfecho }\end{array}$ & $\operatorname{Ins}(3)$ & 5,861 & 0.000 & 0.000 & 1.000 & 0.000 \\
\hline & 4. Insatisfecho & $\operatorname{Ins}(4)$ & 1,993 & 0.000 & 0.000 & 0.000 & 1.000 \\
\hline & 5. Muy insatisfecho & $\operatorname{Ins}(5)$ & 1,559 & 0.000 & 0.000 & 0.000 & 0.000 \\
\hline \multirow[t]{5}{*}{ Estrés $(E s t)$} & 1. Nunca & Est(1) & 3,768 & 1.000 & 0.000 & 0.000 & 0.000 \\
\hline & 2. Casi nunca & Est(2) & 13,256 & 0.000 & 1.000 & 0.000 & 0.000 \\
\hline & 3. Algunas veces & $\operatorname{Est}(3)$ & 6,130 & 0.000 & 0.000 & 1.000 & 0.000 \\
\hline & 4. Frecuentemente & Est(4) & 1,685 & 0.000 & 0.000 & 0.000 & 1.000 \\
\hline & 5. Siempre & Est(5) & 1,473 & 0.000 & 0.000 & 0.000 & 0.000 \\
\hline Tipo & 1. C3 & $\mathrm{TV}(1)$ & 4,783 & 1.000 & 0.000 & & \\
\hline \multirow[t]{2}{*}{ Vehículo (TV) } & 2. T3R3 & $\mathrm{TV}(2)$ & 20,357 & 0.000 & 1.000 & & \\
\hline & 3. T3S2R4 & $\mathrm{TV}(3)$ & 1,172 & 0.000 & 0.000 & & \\
\hline Acceso a la & 0. No tiene & $\mathrm{SS}(0)$ & 9,701 & 1.000 & & & \\
\hline $\begin{array}{l}\text { Seguridad } \\
\text { Social }(S S)\end{array}$ & 1. Si tiene & $\mathrm{SS}(1)$ & 16,611 & 0.000 & & & \\
\hline
\end{tabular}

Fuente: Elaboración propia (Resultados en SPSS).

En la tabla 6, sobre las pruebas de Ómnibus de los coeficientes del modelo, se muestra el incremento del logaritmo neperiano de menos dos veces el logaritmo de la función de verosimilitud y los resultados de la prueba chi-cuadrado que evalúa la hipótesis nula de que todos los coeficientes incluidos en el modelo son cero, dado que $P<0.01$, se considera de manera global que el modelo es estadísticamente significativo. 
Tabla 6. Pruebas ómnibus de coeficientes de modelo.

\begin{tabular}{cccc} 
& $\begin{array}{c}\text { Chi- } \\
\text { cuadrado }\end{array}$ & gl & Sig. \\
\hline Escalón & $7,880.448$ & 12 & 0.000 \\
Bloque & $7,880.448$ & 12 & 0.000 \\
Modelo & $7,880.448$ & 12 & 0.000 \\
gl = grados de libertad. Sig. = Significancia. \\
Fuente: Elaboración propia (Resultados en SPSS).
\end{tabular}

Con la prueba de Hosmer-Lemeshow se confirma la bondad de ajuste total del modelo. El resultado de la prueba es que no se rechaza la hipótesis nula $(P=0.075)$, es decir, que los datos se ajustan al modelo de regresión logística, según Hosmer-Lemeshow. Además, como resultado de esta prueba, en la tabla 7, se puede observar que las coincidencias entre los valores esperados y los observados son altas, tanto de las entregas correctas (Inc_Tpe=0), como de las incorrectas (Inc_Tpe=1).

Tabla 7. Tabla de contingencia para la prueba de Hosmer y Lemeshow.

\begin{tabular}{|c|c|c|c|c|c|}
\hline \multirow{2}{*}{ Paso } & \multicolumn{2}{|c|}{ Inc_Tpe $=.00$} & \multicolumn{2}{|c|}{ Inc_Tpe $=1.00$} & \multirow{2}{*}{ Total } \\
\hline & Observado & Esperado & Observado & Esperado & \\
\hline 1 & 2,632 & $2,631.073$ & 1 & 1.927 & 2,633 \\
\hline 2 & 2,688 & $2,690.695$ & 5 & 2.305 & 2,693 \\
\hline 3 & 2,627 & $2,627.169$ & 3 & 2.831 & 2,630 \\
\hline 4 & 2,624 & $2,622.073$ & 4 & 5.927 & 2,628 \\
\hline 5 & 2,631 & $2,630.514$ & 9 & 9.486 & 2,640 \\
\hline 6 & 2,604 & $2,604.408$ & 12 & 11.592 & 2,616 \\
\hline 7 & 2,630 & $2,618.061$ & 6 & 17.939 & 2,636 \\
\hline 8 & 2,574 & $2,583.427$ & 58 & 48.573 & 2,632 \\
\hline 9 & 2,439 & $2,443.629$ & 196 & 191.371 & 2,635 \\
\hline 10 & 1,071 & $1,068.951$ & 1,498 & $1,500.049$ & 2,569 \\
\hline
\end{tabular}

Fuente: Elaboración propia (Resultados en SPSS).

La tabla de clasificación (tabla 8) muestra que el modelo tiene una especificidad alta (98.5\%) y una sensibilidad media (65.6\%), por lo que el modelo generado a través de la regresión logística ofrece una precisión global de la muestra de $96.3 \%$ en la predicción del evento incumplimiento de entregas a tiempo. 
Entregas tardías o incorrectas en el autotransporte de carga y su relación con las condiciones laborales de los choferes: Un modelo de regresión logística

Tabla 8. Tabla de clasificación.

\begin{tabular}{|c|c|c|c|c|}
\hline \multirow{3}{*}{ Observado } & & \multicolumn{3}{|c|}{ Pronosticado } \\
\hline & & \multicolumn{2}{|c|}{ Inc_Tpe } & \multirow{2}{*}{$\begin{array}{l}\text { Corrección de } \\
\text { porcentaje }\end{array}$} \\
\hline & & .00 & 1.00 & \\
\hline \multirow[t]{2}{*}{ Inc_Tpe } & .00 & 2,4164 & 356 & 98.5 \\
\hline & 1.00 & 616 & 1,176 & 65.6 \\
\hline Porcentaje global & & & & 96.3 \\
\hline
\end{tabular}

a. El valor de corte es .500

Fuente: Elaboración propia (Resultados en SPSS).

En la tabla 9 se reflejan cómo algunas de las variables explicativas están correlacionadas, pero sus efectos independientes sobre la probabilidad de incumplimiento justifican su inclusión en el modelo.

Tabla 9. Matriz de correlaciones.

\begin{tabular}{|c|c|c|c|c|c|c|c|c|c|c|c|c|c|}
\hline Variable & Constante & $T V(1)$ & $T V(2)$ & $T T$ & $S S(1)$ & $\operatorname{Est}(1)$ & $\operatorname{Est}(2)$ & $\operatorname{Est}(3)$ & $\operatorname{Est}(4)$ & $\operatorname{Ins}(1)$ & $\operatorname{Ins}(2)$ & $\operatorname{Ins}(3)$ & $\operatorname{Ins}(4)$ \\
\hline Constante & 1.000 & -.265 & -.144 & -.049 & .043 & -.774 & -.813 & -.859 & -.856 & -.275 & -.312 & -.319 & -.322 \\
\hline$T V(1)$ & -.265 & 1.000 & .546 & -.191 & -.478 & .006 & .008 & .017 & .023 & .013 & .036 & .043 & .037 \\
\hline$T V(2)$ & -.144 & .546 & 1.000 & -.439 & -.214 & .005 & .018 & .033 & .039 & -.010 & .028 & .056 & .046 \\
\hline$T T$ & -.049 & -.191 & -.439 & 1.000 & -.188 & -.002 & -.004 & -.014 & -.016 & -.009 & -.013 & -.047 & -.037 \\
\hline$S S(1)$ & .043 & -.478 & -.214 & -.188 & 1.000 & -.013 & -.022 & -.027 & -.037 & .012 & -.020 & -.029 & -.016 \\
\hline $\operatorname{Est}(1)$ & -.774 & .006 & .005 & -.002 & -.013 & 1.000 & .825 & .852 & .844 & .002 & .003 & .010 & -.008 \\
\hline $\operatorname{Est}(2)$ & -.813 & .008 & .018 & -.004 & -.022 & .825 & 1.000 & .893 & .886 & .004 & .003 & .015 & .008 \\
\hline $\operatorname{Est}(3)$ & -.859 & .017 & .033 & -.014 & -.027 & .852 & .893 & 1.000 & .930 & -.006 & .011 & .062 & .083 \\
\hline Est(4) & -.856 & .023 & .039 & -.016 & -.037 & .844 & .886 & .930 & 1.000 & -.021 & .019 & .076 & .097 \\
\hline $\operatorname{Ins}(1)$ & -.275 & .013 & -.010 & -.009 & .012 & .002 & .004 & -.006 & -.021 & 1.000 & .616 & .548 & .517 \\
\hline $\operatorname{Ins}(2)$ & -.312 & .036 & .028 & -.013 & -.020 & .003 & .003 & .011 & .019 & .616 & 1.000 & .590 & .564 \\
\hline $\operatorname{Ins(3)}$ & -.319 & .043 & .056 & -.047 & -.029 & .010 & .015 & .062 & .076 & .548 & .590 & 1.000 & .539 \\
\hline $\operatorname{Ins}(4)$ & -.322 & .037 & .046 & -.037 & -.016 & -.008 & .008 & .083 & .097 & .517 & .564 & .539 & 1.000 \\
\hline
\end{tabular}

Fuente: Elaboración propia (Resultados en SPSS).

Finalmente, durante el cálculo de las probabilidades correspondientes a los datos pronosticados del modelo [variable P(Inc_Tpe)], se observó que la mayor probabilidad de incumplimiento en las entregas a tiempo (97.18\%) se obtiene cuando se utiliza el vehículo más grande T3S2R4 
$[T V(3)=1]$, los choferes no tienen seguridad social $(S S=0)$, el estrés y la insatisfacción son altas $[\operatorname{Est}(5)=1$ e $\operatorname{Ins}(5)=1]$ y el tránsito es largo (TT=36 horas). Por el contrario, la menor probabilidad de incumplimiento en las entregas a tiempo (0.23\%) se obtiene cuando el vehículo es el de menor capacidad $[\operatorname{TV}(1)=1]$, los choferes tienen seguridad social [SS=1], el tiempo de tránsito es corto $[\mathrm{TT}=3]$ y el estrés y la insatisfacción tienen bajos valores $[\operatorname{Est}(1)=1, \operatorname{Ins}(1)=1]$.

\section{$\underline{\text { Discusión }}$}

Algunos estudios sobre conductores han demostrado, que el estrés, afecta la productividad y el desempeño de los trabajadores del volante (Bathija et al., 2014; Lámbarry et al., 2016). Así, no es de extrañar que el estrés se haya incluido como uno de los factores en el modelo y que, en la medida en que incrementa, también aumenta la probabilidad de incumplimiento en las entregas a tiempo. Sin embargo, dado que el estrés es provocado por jornadas que no atienden de manera adecuada las necesidades de descanso, la falta de capacitación para conducir sus vehículos o por las condiciones laborales en las que se desenvuelven los trabajadores (Lámbarry et al., 2016), tratar de reducir el nivel de estrés es una tarea complicada, ya incluye prácticamente todas las categorías de las condiciones laborales.

No obstante, se sabe que parte importante de las condiciones laborales de los conductores se encuentran determinadas por las características del vehículo en el que desempeñan sus actividades y que, a su vez, las características del vehículo influyen de manera importante en la satisfacción de los conductores (Ordaz et al., 2007). Así, dado que en México 88\% de la flota vehicular tiene una antigüedad de más de veinte años (DGAF, 2015); es evidente el atraso en condiciones de ergonomía, confort, ruido, seguridad y dispositivos tecnológicos que facilitan la conducción; por lo que los vehículos antiguos no sólo afectan las condiciones laborales y la satisfacción de los conductores, sino que ponen en constante desventaja la competitividad de las empresas.

Además, la satisfacción comprende otras dimensiones adicionales a las concernientes a los vehículos; tales como las exigencias laborales, cuestiones organizacionales, clima laboral, la remuneración, la naturaleza de las tareas realizadas y hasta situaciones personales. En este estudio, se evaluó la satisfacción de desde dos perspectivas: 1) La insatisfacción subjetiva, que evalúa de manera general los sentimientos y emociones de los conductores (variable Ins); y 2) La satisfacción con el ingreso (variable $S I$ ), que evalúa bienestar o placer en relación al dinero que 
perciben los conductores por su trabajo. Así, en el modelo se incluyó la insatisfacción subjetiva, ya que resultó significativa $(\mathrm{P}<0.01)$, y se excluyó la variable relacionada a la satisfacción con el ingreso, que no fue significativa $(\mathrm{P}=0.735)$.

En general, se deduce que los choferes se encuentran satisfechos con sus ingresos, aunque no con otras condiciones laborales que se relacionan directamente con los incumplimientos de las entregas a tiempo, por lo que se puede afirmar que a mayores niveles de insatisfacción mayor es el riesgo para incumplir en las entregas a tiempo.

Por su parte, aunque la variable de satisfacción en el ingreso no fue significativa y, por tanto, no fue incluida en el modelo, se conoce que el ingreso de los choferes se relaciona con la experiencia y la habilidad de conducir vehículos más grandes. Así, por ejemplo, un conductor de vehículos con doble remolque obtiene más ingresos, que el de un remolque y, este a su vez, mayores ingresos que los de un camión unitario. No obstante, el modelo muestra que los vehículos que mueven mayor cantidad de carga (con dos remolques T3S2R4), tienen mayor probabilidad de incumplimiento en las entregas a tiempo.

Otros estudios indican que la razón de tasas de morir en un percance vial aumenta con el tamaño del vehículo, y se incrementa hasta 3.42 veces para los vehículos más pesados (Berrones et al., 2018). Si bien, el modelo no profundiza en los accidentes, aunque si los contempla como incumplimiento en las entregas a tiempo, los resultados concuerdan en el riesgo que el tamaño de los vehículos (variable $T V$ ) impone en la cadena de suministro.

Dado que los vehículos más grandes son más eficientes en cuestiones de costos, y utilizar las diferentes configuraciones vehiculares depende de la planeación y necesidades específicas -como el tamaño del pedido o condiciones del mercado- no es factible, sin previas consideraciones, utilizar vehículos más pequeños; por lo que se debe promover la capacitación y la promoción de la seguridad vial, de tal manera que, progresivamente, los conductores tengan nuevas habilidades que compensen las dimensiones de los vehículos y disminuyan los riesgos en la cadena de suministro.

Aunque el modelo planteado, para calcular la probabilidad de incumplimiento en las entregas, no contempla de forma directa la jornada de trabajo, los días laborados por semana o el tiempo de descanso de los conductores; sí incorpora dentro de las variables con significancia estadística el tiempo de tránsito programado, que es parte del tiempo de trabajo, y calcula su mayor probabilidad de incumplimiento $(97.18 \%)$ en las entregas programadas a 36 horas 
$(T T=36)$. Durante ese tiempo, el chofer realiza la entrega desde el origen hasta el destino, está presente durante la carga y descarga, se alimenta, descansa, duerme y recorre poco más de mil kilómetros.

En México, es común que los choferes del autotransporte de carga trabajen por destajo y bajo una laxa regulación de las horas de conducción que, además, no contempla las horas de trabajo fuera del volante. A pesar de que las largas jornadas de los conductores puede causar irritabilidad, fatiga física y mental, somnolencia excesiva o insomnio, falta de atención en el trabajo y accidentes de tránsito (Chun-Chieh et al., 2010), se sabe que en este país es una práctica común que los choferes laboren hasta 76 horas por semana sin el debido descanso (Vargas, 2013). En consecuencia, los efectos del tiempo de tránsito y las largas horas de trabajo, no sólo ponen en riesgo a los procesos logísticos a través de los incumplimientos de las entregas a tiempo, sino que están asociadas con efectos adversos para la salud, como las enfermedades cardiovasculares, la diabetes o la jubilación por discapacidad. (Van-Der-Beek, 2012).

Asimismo, a pesar de que varios estudios alrededor del mundo señalan que la actividad de conducir se encuentra entre las profesiones que tienen mayores padecimientos y factores de riesgos asociados a su ocupación (Bigert, 2004; Shin et al., 2013), la condición subjetiva de enfermo o no enfermo (Variable Enf) no demostró significancia estadística $(\mathrm{P}=0.276)$ y no fue considerada para el cálculo de la probabilidad de incumplimiento de transporte.

Por su parte, la derechohabiencia de Seguridad Social (Variable $S S$ ) si tuvo significancia estadística $(\mathrm{P}<0.01)$ y fue considerada para el cálculo de probabilidad de incumplimiento. Sin embargo, el porcentaje de choferes que disponen de estas prestaciones es alto en comparación con el registro del total nacional de los trabajadores de tránsito terrestre, 63.12\% para el caso de estudio contra 39.95\% de los conductores registrados en la encuesta nacional de ocupación y empleo (INEGI, 2017). Es importante señalar que, para este caso de estudio, la cifra se ve influida por la cantidad de empresas con más de treinta vehículos, $17.31 \%$ contra $2.2 \%$ en el total nacional (DGAF, 2015); ya que, por el contrario de las empresas de tipo hombre-camión, las empresas de mayor tamaño sí ofrecen estas prestaciones a sus empleados.

En consecuencia, tener el beneficio de la seguridad social $(S S=1)$, representa que el conductor es acreedor a una serie de privilegios, en los que se incluye el acceso a los servicios de salud, vacaciones, aguinaldo, sistemas de pensiones, entre otros; que garantizan cierta conformidad en las condiciones laborales. Así, a pesar de que, en muchos casos, como estrategia 
de evasión de cuotas patronales, la seguridad social sólo contempla el salario mínimo y no la remuneración real del conductor -por lo que, en caso de jubilación o incapacidad temporal o definitiva, sólo perciben una mínima parte del ingreso habitual- el carecer de la seguridad social $(S S=0)$ es un factor que participa en el cálculo de la mayor probabilidad de incumplimiento en las entregas a tiempo.

Finalmente, en comparación con otros modelos de regresión logística, y a pesar de que tienen diferentes objetivos, se puede observar que, para explicar algunos comportamientos, se incluyen algunas variables similares a las encontradas en el modelo para predecir los incumplimientos en las entregas de transporte. Así, Martínez et al. (2015) para explicar la calidad de vida laboral, incluyó variables de estabilidad laboral, carga física, salario y algunos otros estresores. Destaca la similitud de las variables relativas al estrés y a la carga física; también consideradas, a través de las variables Est y TT, en los resultados del modelo de incumplimiento de trasporte.

Además, al igual que en el modelo propuesto, ni en el trabajo de Martínez et al. (2015) ni en el de Villar et al. (2015) resulta significancia estadística para la variable remuneración, a pesar de que el ingreso se correlaciona positivamente con las condiciones subjetivas de trabajo; por lo que puede afirmar que el ingreso no es tan importante como otras condiciones laborales para el comportamiento de los trabajadores.

Por su parte, Villar et al. (2015) tampoco encontró significancia estadística en la región donde laboran sus empleados $y$, al igual que la variable zona de trabajo $(Z T)$, fue excluida del modelo final. Sin embargo, indica que en la satisfacción influye la autonomía de los trabajadores y; dado que, en el modelo de cumplimiento de transporte, la variable propiedad del vehículo $(P V)$ representa la autonomía en el trabajo, es posible considerar cierta contradicción entre ambos estudios. No obstante, el estudio de comparación se refiere a empleados administrativos, mientras que el actual se basa en choferes; y la actividad de conducir ya lleva implícita cierta autonomía en el trabajo.

Así, a pesar de los diferentes objetivos de las investigaciones, los modelos de regresión logística, parecen ser una buena alternativa, para ser utilizados como metodologías para explicar y predecir las relaciones entre las condiciones laborales y el comportamiento de los trabajadores. 


\section{Conclusiones}

A pesar de que los diferentes grupos de conductores comparten riegos y exigencias derivadas de su proceso de trabajo, sus condiciones laborales varían de acuerdo a diferentes factores determinados por aspectos tecnológicos, sociales, culturales, económicos y de regulación, tanto en materia laboral como de tránsito, por lo que algunas otras variables, no incluidas en el modelo, podrían resultar relevantes.

No obstante, las cinco variables utilizadas para el cálculo de la probabilidad de incumplimiento pueden ser consideradas dimensiones que engloban otros factores y se relacionan con otros padecimientos en las condiciones laborales y de salud, tanto física como mental. Así, por ejemplo, la variable $T E$ (tamaño de empresa) se asocia a la edad de la flota vehicular; ya que las empresas de mayor tamaño tienen vehículos con modelos menores a seis años, mientras que las de tipo hombre-camión tienen vehículos con periodos de utilización superiores a los veinte años.

En suma, para extrapolar el modelo a otro tipo de industrias, es necesario incluir mayor cantidad de variables (demográficas, de salud, de trabajo y de organización, entre otras), para descartar omisiones de factores o características relevantes, propias de ciertas industrias o productos, que puedan afectar las entregas a tiempo y, por lo tanto, a la cadena de suministro.

Por otro lado, en el modelo para el cálculo de la probabilidad de incumplimiento, dos de las variables fueron declaraciones de los conductores, es decir, son de carácter subjetivo. Se recomienda que se busquen métodos y pruebas objetivas para medir y reducir el nivel de estrés, la insatisfacción laboral y el resto de las variables subjetivas.

Finalmente, a pesar de las limitaciones del modelo, los resultados muestran una herramienta prometedora para proporcionar interpretaciones significativas que pueden utilizarse para futuras mejoras en el desempeño de las empresas del autotransporte de carga. De esta forma, se demuestra la importancia de las condiciones laborales y la forma en que influyen en los incumplimientos de las entregas a tiempo del autotransporte de carga; por lo que el modelo será utilizado para crear un modelo conceptual, que generalice en cualquier tipo de industria, el impacto de las condiciones laborales en las entregas a tiempo y, por lo tanto, en la cadena de suministro. 
Entregas tardías o incorrectas en el autotransporte de carga y su relación con las condiciones laborales de los choferes: Un modelo de regresión logística

\section{$\underline{\text { Agradecimientos }}$}

A la empresa de manufactura de sistemas de construcción ligera, y la Universidad Autónoma de la Ciudad de México, que hicieron posible este documento.

\section{$\underline{\text { Referencias }}$}

Al-Ghamdi, A. S. (2002). Using logistic regression to estimate the influence of accident factors on accident severity. Accident Analysis \& Prevention, 34(6), 729-741. https://doi.org/10.1016/S0001-4575(01)00073-2

Álvarez-Cáceres, R. (2007). Estadística aplicada a las ciencias de la salud. Madrid: Díaz de Santos.

Apostolopoulos, Y., Sönmez, S., Shattell, M. M., \& Belzer, M. (2010). Worksite-induced morbidities among truck drivers in the United States. AAOHN Journal: Official Journal of the American Association of Occupational Health Nurses, 58(7), 285-296. https://doi.org/10.3928/08910162-20100625-01

Ballou, R. H. (2004). Business logistics/supply chain management: Planning, organizing, and controlling the supply chain (5. ed.). Upper Saddle River, NJ: Pearson Prentice Hall.

Bathija, G., Bant, D., Itagimath, S., Lokare, L., Godbole, M., Nekar, M., Shidaraddi, K., Kurigodivar, M., Reddi, K. (2014). A study on stress among government city bus drivers in Hubli. International Journal of Biomedical Research, 5(2), 102. https://doi.org/10.7439/ijbr.v5i2.503

Berrones, L. (2017). Choferes del autotransporte de carga en México: investigaciones sobre condiciones laborales y la cadena de suministro. Revista Transporte y Territorio, 1(17), 252-267.

Berrones, L., Cano, P., Sánchez, D., \& Martínez, J. (2018). Lesiones, enfermedades y accidentes de trabajo de los conductores del autotransporte de carga en México. Forthcoming.

Bigert, C. (2004). Time trends in the incidence of myocardial infarction among professional drivers in Stockholm 1977-96. Occupational and environmental medicine, 61(12), 987991. https://doi.org/10.1136/oem.2004.012781

Blanquart, C., \& Burmeister, A. (2009). Evaluating the performance of freight transport: A service approach. European Transport Research Review, 1(3), 135-145. https://doi.org/10.1007/s12544-009-0014-5 
Chopra, S., \& Meindl, P. (2016). Supply chain management: Strategy, planning, and operation (Sixth Edition). Boston: Pearson.

Chun-Chieh, C., Li-Jie, S., Yu-Ling, L., Kuan-Yeng, T., Kwan-Yu, C., Chih-Jung, Y., ShiuanChih, C., Ruey-Hong, W. (2010). Shift work and arteriosclerosis risk in professional bus drivers. Annals of epidemiology, 20(1), 60-66. https://doi.org/10.1016/j.annepidem.2009.07.093

DGAF. (2015). Estadística Básica del Autotransporte Federal 2015. México: Dirección General de Autotransporte Federal; Secretaría de Comunicaciones y Transportes.

Fiuza, M. D., \& Rodríguez, J. (2000). La regresión logística: Una herramienta versátil. Nefrología, 20(6), 495-500.

Garcia, L., Dias, C., Pereira, L., Cesar, M., Romero, D., \& Leyton, V. (2016). Truck drivers’ traffic accidents in the State of São Paulo: prevalence and predictors. Ciencia \& saude coletiva, 21(12), 3757-3767. https://doi.org/10.1590/1413-812320152112.11182015

Hilakivi, I., Veilahti, J., Asplund, P., Sinivuo, J., Laitinen, L., \& Koskenvuo, K. (1989). A sixteen-factor personality test for predicting automobile driving accidents of young drivers. Accident; analysis and prevention, 21(5), 413-418.

IMT. (2015). Manual estadístico del sector transporte 2015. Sanfandila, Qro.: Instituto Mexicano del Transporte (IMT).

INEGI. (2017). Encuesta Nacional de Ocupación y Empleo (ENOE). Población de 15 años y más de edad: Población ocupada. Retrieved from http://www.beta.inegi.org.mx

Lámbarry, F., Trujillo, M., \& Cumbres, C. (2016). Stress from an administrative perspective in public transport drivers in Mexico City: Minibus and metrobus. Estudios Gerenciales. Advance online publication. https://doi.org/10.1016/j.estger.2016.02.003

Magee, C., Stefanic, N., Caputi, P., \& Iverson, D. (2011). Occupational factors and sick leave in Australian employees. Journal of occupational and environmental medicine, 53(6), 627632. https://doi.org/10.1097/JOM.0b013e31821df4cf

Martínez, L., Oviedo, O., \& Luna, C. (2015). Impact of working conditions on the quality of working life: Case manufacturing sector colombian Caribbean Region. DYNA, 82(194), 194-203. https://doi.org/10.15446/dyna.v82n194.49293

Mehrjoo, S., \& Bashiri, M. (2013). An application of principal component analysis and logistic regression to facilitate production scheduling decision support system: An automotive 
Entregas tardías o incorrectas en el autotransporte de carga y su relación con las condiciones laborales de los choferes: Un modelo de regresión logística

industry case. Journal of Industrial Engineering International, 9(1), 1-12. https://doi.org/10.1186/2251-712X-9-14

Müller, C. F., Monrad, T., Biering-Sørensen, F., Darre, E., Deis, A., \& Kryger, P. (1999). The influence of previous low back trouble, general health, and working conditions on future sick-listing because of low back trouble: A 15-year follow-up study of risk indicators for self-reported sick-listing caused by low back trouble. Spine, 24(15), 1562-1570. https://doi.org/10.1097/00007632-199908010-00010

Nogueira, G., Carvalho, D., Siqueira, C., Borges, P., de Almeida, W., Sisinno, L., \& Landmann, C. (2016). Alcohol abuse and involvement in traffic accidents in the Brazilian population, 2013. Ciencia \& saude coletiva, 21(12), 3777-3786. https://doi.org/10.1590/1413812320152112.25692015

Ordaz, E., Maqueda, J., Silva, A., Asúnsolo, Á., Prieto, D., \& Olmedo, O. (2007). Salud y Condiciones de Trabajo en el Transporte de Mercancías por Carretera. Madrid, España: Instituto de Salud Carlos III.

Paydar, S., Endut, I. R., \& Lajevardi, A. (2013). Environmental determinants of RFID adoption in retail supply chain, a binary logistic regression analysis. In IEEE International Conference on RFID-Technologies and Applications, RFID-TA 2013. Malaysia: IEEE. https://doi.org/10.1109/RFID-TA.2013.6694547

Pérez, C. (2004). Técnicas de análisis multivariante de datos: Aplicaciones con SPSS. Madrid: Pearson Prentice Hall.

Sánchez-Gómez, M. G. (2008). Cuantificación y generación de valor en la cadena de suministro extendida. León: Del Blanco.

Shin, S. Y., Lee, C. G., Song, H. S., Kim, S. H., Lee, H. S., Jung, M. S., \& Yoo, S. K. (2013). Cardiovascular Disease Risk of Bus Drivers in a City of Korea. Annals of Occupational and Environmental Medicine, 25(1), 34. https://doi.org/10.1186/2052-4374-25-34

Sluis, S., \& Giovanni, P. de. (2016). The selection of contracts in supply chains: An empirical analysis. Journal of Operations Management, 41, 1-11. https://doi.org/10.1016/j.jom.2015.10.002

Suwazono, Y., Dochi, M., Kobayashi, E., Oishi, M., Okubo, Y., Tanaka, K., \& Sakata, K. (2008). Benchmark duration of work hours for development of fatigue symptoms in Japanese workers with adjustment for job-related stress. Risk analysis: an official publication of the 
Society for Risk Analysis, 28(6), 1689-1698. https://doi.org/10.1111/j.15396924.2008.01107.x

Van-Der-Beek, A. (2012). World at work: truck drivers. Occupational and environmental medicine, 69(4), 291-295. https://doi.org/10.1136/oemed-2011-100342

Vargas, J. M. (2013). Kilómetro a kilómetro guachicoleándose la vida. El caso del hombre camión en una empresa Queretana. Universidad Autónoma de Querétaro (UAQ), Quéretaro.

Villar, E., Delgado, J., \& Barrilao, P. (2015). Job Satisfaction Among Spanish Tax Administration Employees: A Logistic Regression Analysis. Journal of Labor Research, 36(2), 210-223. https://doi.org/10.1007/s12122-015-9202-3

Zhu, Y., Xie, C., Sun, B., Wang, G. J., \& Yan, X. G. (2016). Predicting China's SME Credit Risk in Supply Chain Financing by Logistic Regression, Artificial Neural Network and Hybrid Models. Sustainability, 8(5), 433. https://doi.org/10.3390/su8050433 